\title{
Total laparoscopic pancreaticoduodenectomy
}

\author{
Alessandro Paniccia, Brandon Chapman, Barish H. Edil, Richard D. Schulick \\ Department of Surgery, University of Colorado Anschutz Medical Campus, Aurora, CO, USA \\ Correspondence to: Richard D. Schulick, MD, MBA. Department of Surgery, University of Colorado Anschutz Medical Campus, 12631 East 17th Ave., \\ Aurora, CO, USA. Email: richard.schulick@ucdenver.edu.
}

\begin{abstract}
Total laparoscopic pancreaticoduodenectomy (TLP) represents perhaps one of the most challenging abdominal operations. This procedure is gaining popularity in recent years, mostly due to the numerous reports underling its safety and feasibility in the setting of several benign and malignant pancreatic pathologies. Minimal-invasive pancreatic surgery is rapidly becoming a reality in many centers around the globe and its benefits, compared to the traditional open approach, have been extensively proven in the setting of distal pancreatic resection. The many advantages of a laparoscopic approach, such as the improved visual magnification, the theoretical improved tissue exposure, and the potential for a more delicate manipulation of tissues are reasonably expected to be applicable to other pancreatic procedures including TLP. Herein we describe a technique for a TLP; we provide some suggestions on patient selection, pre-operative preparation, equipment, postoperative management, and finally discuss some of the most common pitfalls encountered during the procedure.
\end{abstract}

Keywords: Laparoscopic pancreaticoduodenectomy; Whipple; minimally invasive pancreatic surgery; pancreas

Received: 10 May 2016; Accepted: 29 June 2016; Published: 02 August 2016.

doi: 10.21037 /jovs.2016.07.13

View this article at: http://dx.doi.org/10.21037/jovs.2016.07.13

\section{Introduction}

Total laparoscopic pancreaticoduodenectomy (TLP) has remained a topic of controversy in the surgical literature during the last two decades. TLP was first reported in 1994 by Gagner and Pomp, who performed the procedure in the setting of chronic pancreatitis (1). Since then, several single institution series reported on the safety and feasibility of TLP in the setting of several benign and malignant pancreatic pathologies (2-4). The available literature mainly focuses on two key aspects of TLP, one being the learning curve necessary to master the procedure and the other being the comparison of overall outcomes between TLP and open pancreaticoduodenectomy (OPD) (5-7). The numerous studies that have focused on TLP learning curve have identified at least three phases: a slow difficult beginning, followed by a rapid improvement that culminates in a plateau phase characterized by a slow but continuous improvement (5-7). Moreover, most studies comparing TLP to OPD suggest that when TLP is performed in center of expertise the oncologic outcome, the complication rate, and the mortality rate are similar to OPD. Furthermore, TLP appears to be associated with decreased blood loss and hospital stay despite being associated with longer operative time (7-11).

Minimally invasive approaches to pancreatic surgery represent the most recent technical innovation in the field of pancreatic surgery and range from a laparoscopic assisted procedure, to a total laparoscopic or robotic approach.

The benefits of a minimal invasive pancreatic surgery have been extensively recognized for distal pancreatic resection and include reduced blood loss, shorter hospitalization, and reduced overall complication rates compared with the standard open approach (12-15). However, the definitive benefits of a minimally invasive approach (laparoscopic or robotic) to pancreaticoduodenectomy (Whipple procedure) continue to be debated $(10,16-18)$. Nevertheless, it appears reasonable to speculate that some of the advantages seen with the use of a minimally invasive approach to distal pancreatectomy may be applicable to a TLP.

Herein we describe a technique for a TLP; we provide some suggestions on patient selection, pre-operative 


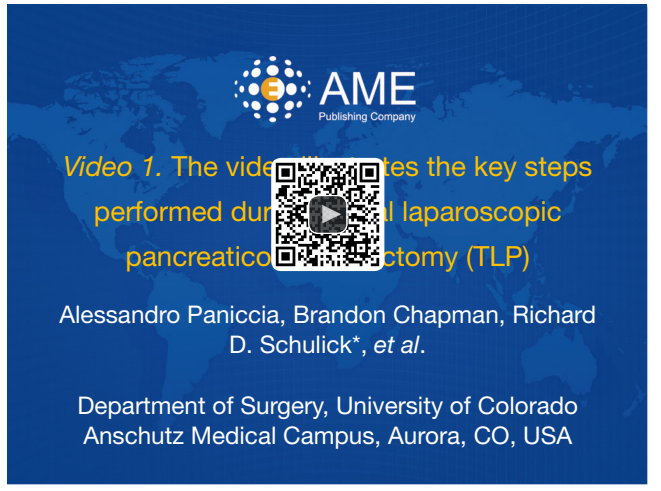

Figure 1 The video illustrates the key steps performed during a total laparoscopic pancreaticoduodenectomy (TLP) (20).

Available online: http://www.asvide.com/articles/1057

preparation, equipment, postoperative management, and finally discuss some of the most common pitfalls encountered during the procedure.

\section{Patient selection and workup}

Most patients with pancreatic, ampullary, or biliary pathologies who require a pancreaticoduodenectomy are eligible for a laparoscopic approach. One limitation is represented by patients with locally advanced pathologies (i.e., locally advanced pancreatic adenocarcinoma) with involvement of the mesenteric vasculature due to the inherent technical difficulties represented by the need for laparoscopic vascular resection and reconstruction (although a few specialized centers occasionally offer TLP in this setting) (11,19).

The authors routinely obtain preoperative multi-slice pancreas specific triple-phase (i.e., arterial, late arterial, and venous phase) computed tomography in order to properly evaluate the pancreatic gland and its spatial relation with the surrounding organs and vasculature; particular attention is given to the evaluation for any aberrant anatomy (e.g., replaced or accessory hepatic vasculature).

Additional imaging is dictated by the particular pancreatic pathology that is being addressed, and can vary from endoscopic retrograde cholangiopancreatography with or without pancreatic duct brushing, endoscopic pancreatic ultrasound with or without fine needle aspiration, and magnetic resonance imaging.

Preoperative laboratory tests are routinely obtained and include a complete blood cell count, a complete metabolic panel (CMP), a coagulation profile, and in case of a patient with known preexisting diabetes glycated hemoglobin level is assessed; specific tumors markers are obtained based on the pathology being treated.

\section{Pre-operative preparation}

Patients selected to undergo a TLP meet preoperatively with a nutritionist and a glucose management expert, this provides an initial overview on the life style and diet adjustments that are necessary following the procedure. Furthermore, this initial encounter represents an opportunity for the patient to become familiar with the available insulin treatment regimens and glycemic monitoring strategies, should the need arise.

\section{Procedure}

This section summarizes the key steps performed during a TLP as illustrated in the multimedia file supplement associated with this manuscript (Figure 1).

\section{Dissection phase}

The patient is placed supine on the operating table and care is taken to properly secure the patient with a thigh belt to the operating table. It is paramount to ensure proper patient positioning and stability, as the table will be tilted at different stages of the procedure to help with organ exposure during tissue dissection and reconstruction. The upper extremity bony prominences are covered with soft pads; both arms are extended to no more than a $60^{\circ}$ degree (to avoid injury to the brachial plexus).

The surgeon is positioned on the left side of the patient, the first assistant is positioned on the right side of the patient, and the second assistant stands on the left side of the patient, next to the surgeon. However, throughout the case operating is done from both sides of the patient depending on what is being done.

The procedure can be performed using five trocars, including a Hassan optical trocar, two $12 \mathrm{~mm}$ trocars, and two $5 \mathrm{~mm}$ trocars.

The Hassan optical trocar is positioned at the umbilicus (to be used for a $10 \mathrm{~mm} 30^{\circ}$ or $45^{\circ}$ angled laparoscope), two $12 \mathrm{~mm}$ trocars are placed along both left and right hemiclavicular line approximately $2 \mathrm{~cm}$ below the rib cage (these are the two main working ports), one additional $5 \mathrm{~mm}$ trocars is placed on the right side of the umbilicus (to provide lateral traction as needed) and an additional $5 \mathrm{~mm}$ 
trocar can be placed on the left of the umbilicus as needed.

Once the abdominal cavity is accessed, the abdomen is first explored then attention is turned to the identification of the lesser sac. An ultrasonic dissector is used to divide the gastrocolic ligament, below the gastroepiploic vessels, allowing access to the retroperitoneal area and ultimately leading to direct visualization of the pancreas.

Subsequently the assistant provides cephalad traction on the stomach (by grasping the stomach antrum or body with an atraumatic laparoscopic grasper); this will facilitate the identification of any adhesions present between the posterior surface of the stomach and the anterior surface of the pancreas. These adhesions can then be sharply divided gaining full exposure of the anterior surface of the pancreas.

The dissection of the porta hepatis is initiated and the gastroduodenal artery (GDA) lymph node is identified and removed. The removal of the GDA lymph node allows visualization of the GDA take-off that is isolated and skeletonized, care is taken to avoid avulsion of the superior anterior pancreaticoduodenal artery.

The GDA can now be ligated; the authors prefer a suture ligation of the proximal GDA that is additionally reinforced with two medium surgical clips, proximally and distally, prior to its sharp division. Care is taken to verify that the GDA is properly skeletonized and removed of all surrounding soft tissue in order to ensure full ligation with the surgical clips.

Attention is then turned to the inferior pancreatic border and care is taken to identify the superior mesenteric vein (SMV). Blunt dissection is carried on along the SMV anterior surface, progressively separating the posterior aspect of the pancreatic neck from the SMV and eventually leading to the identification of the confluence between the SMV vein and the splenic vein. During this step, the laparoscopic approach offers a magnified visualization of the "tunnel" created between the pancreatic neck and the SMVsplenic vein confluence that is a clear advantage compared to a traditional open procedure.

The hepatic flexure and the transverse colon are mobilized inferiorly after division of the colohepatic peritoneum exposing the second and third portion of the duodenum. An extended Kocher maneuver is performed to allow for medialization of the duodenum and the plane between the duodenum and the retroperitoneum is identified and dissected using either blunt or energy dissection to allow for the identification of the inferior vena cava, the aorta, and the superior mesenteric artery.
The gallbladder is identified, the Calot's triangle is exposed, and the cystic duct and the cystic artery are dissected and doubly ligated with surgical clips prior to being sharply divided. A cholecystectomy is then completed in a standard laparoscopic fashion and the dissected gallbladder is placed in the right abdomen for later removal.

The stomach can then be transected just proximal to the pylorus using a laparoscopic stapling device (the pylorus should be clearly identified prior to the transection in order to avoid accidently stapling across the pylorus). The gastric remnant can now be mobilized into the left upper abdomen allowing for improved exposure of the pancreas.

The pancreatic neck is then divided along the previously created pancreatic tunnel (with the use of electrocautery) and the pancreaticoduodenal arteries are controlled (with the use of an energy device) for hemostasis. The pancreatic duct is identified and an appropriately sized pediatric feeding tube (usually ranging from 4 to 8 French) is inserted in the pancreatic duct; this will function as a temporary stent and will aid with the subsequent reconstruction.

The common bile duct is then identified, dissected free from the surrounding tissues and its proximal aspect is secured with a surgical bulldog clamp, this will avoid spillage of bile during the remaining steps of the procedure. An energy device is then used to transect the common bile duct approximately 2 to $3 \mathrm{~cm}$ above the superior pancreatic border.

The authors use a laparoscopic stapler to divide the jejunum to $50 \%$ of its width at site chosen for the future definitive transection; the division of only half of the jejunum allows for easier rotation of the jejunum through the ligament of Treitz and under the mesenteric vessels. Alternatively, as shown in the video, the jejunum can be completely transected and the two jejunal free ends can be held together by a stay suture that will eventually allow for easy jejunal rotation under the mesenteric vessels.

The ligament of Treitz is identified and mobilized from its retroperitoneal attachments, using blunt dissection and an energy device. Once the dissection is completed, the duodenum and the jejunum can be safely rotated under the mesenteric vessels. A window is created in the mesentery, approximately 15 to $20 \mathrm{~cm}$ distal to the duodenojejunal flexure, and the jejunal vascular arcades are serially divided with the use of an energy device.

Attention is then turned to the pancreatic neck with the ultimate goal to expose and to dissect free the uncinate process. The assistant applies gentle cephalad and lateral 
traction to the pancreatic head (toward the patient's right), this allows the surgeon to perform a blunt dissection along the SMV-portal vein confluence achieving complete separation between these structures and the posterior surface of the remaining pancreas.

At this stage, the uncinate process can be dissected free from the superior mesenteric artery using an energy device, however, occasionally it will require clips or suture ligature. A laparoscopic suctioning device can be used to gently retract the SMV laterally (toward patient's left side) allowing for complete visualization of the attachment between the SMA and the uncinate process. It is paramount to visualize both vessels (SMV and SMA) simultaneously during this delicate dissection in order to avoid catastrophic venous or arterial injuries.

Ultimately, the jejunum can be completely transected (at the site of the previous partial transection) using a laparoscopic stapling device.

This final step completes the dissection portion of the procedure and the specimens, including the previously dissected gallbladder, can be safely removed using a laparoscopic endo-bag and extracted through the umbilical port site.

\section{Reconstruction phase}

The reconstruction commences with the creation of a duct to mucosa pancreaticojejunostomy. The free end of the jejunum is brought in close proximity to the pancreatic remnant in preparation for an end to side, duct to mucosa pancreaticojejunostomy. The anastomosis begins with the construction of the posterior anastomotic row, which is fashioned using a single-layered running 4-0 barbing suture that eliminates the need for knots to secure suture lines. Then, a $2-3 \mathrm{~mm}$ jejunotomy is made to allow for a duct to mucosa anastomosis. After securing the pancreatic duct to the jejunal mucosa with a $5-0$ synthetic nonabsorbable suture, the pancreatic duct stent is passed through the jejunal defect and a duct to mucosa anastomosis is completed using five or six additional 5-0 synthetic nonabsorbable sutures in an interrupted fashion. Finally, a single-layered running anastomosis is performed using a barbed suture on the anterior side.

The completion of a pancreaticojejunostomy is followed by the creation of an end-to-side choledochojejunostomy. The previously transected CBD is gently dilated with the use of a laparoscopic Maryland dissector instrument (by gentle separation of the instrument jaws) to allow for an easier anastomosis. Then, a jejunotomy is performed on the antemesenteric portion of the free-jejunal-end with the use of a laparoscopic electrocautery; this site is again gently dilated with a laparoscopic Maryland dissector to approximately match the size of the previously transected choledocho. An end-to-side duct-to-mucosa choledochojejunostomy anastomosis is performed using interrupted 4-0 synthetic absorbable sutures; the posterior row of the anastomosis is fashioned first and usually requires 3 to 4 interrupted sutures. Once the posterior row of the anastomosis is completed, a 6 to 8 French silicone tube (usually a pediatric feeding tube) can be customized to serve as a temporary biliary stent and inserted through the anterior opening of the choledochojejunostomy, this is followed by completion of the anterior row of the anastomosis in a similar fashion.

To minimize the tension of the choledochojejunostomy anastomosis, the authors routinely anchor the free-end of the jejunal limb to the hilar plate using one or two interrupted 3-0 synthetic absorbable sutures.

The mesenteric defect can now be closed with interrupted 3-0 silk sutures.

A jejunal loop is brought closer to the gastric remnant in preparation for an antecolic gastrojejunostomy, two 3-0 silk sutures are placed proximally and distally along the length of the future anastomosis to serve as anchoring sutures (stay-suture) so to facilitate the alignment of the jejunal segment to the stomach remnant. The assistant can now hold the tail of the proximal anchoring suture up toward the abdominal wall while applying gentle tension to the distal jejunal limb, at the same time the surgeon applies gentle cephalad tension to the stomach remnant; this maneuver stabilizes the gasto-jejunal unit and two enterotomies (a gastrotomy and a jejunotomy) can be easily created using an energy device.

A gastrojejunotomy is then completed using a stapling device; the resulting common enterotomy defect is closed with interrupted 3-0 silk sutures.

Finally the abdomen is explored for evidence of bleeding, bile leakage, or remaining enterotomy defects; one surgical drain is placed posteriorly to the pancreaticojejunostomy and one anterior to the choledochojejunostomy. The abdominal wall fascial defects are finally closed with the use of a Carter-Thomason needle suture passer.

\section{Equipment card}

The key surgical instruments and supplies necessary to 
Table 1 TLP equipment card (includes key components)

\begin{tabular}{|c|c|}
\hline Item name & Quantity \\
\hline $5 \mathrm{~mm} 0^{\circ}$ and $30^{\circ}$ laparoscopes & 1 \\
\hline $10 \mathrm{~mm} 0^{\circ}$ and $45^{\circ}$ laparoscopes & 1 \\
\hline Endoscopic kittner & 1 \\
\hline Harmonic scalpel $A C E^{\circledR}$ laparoscopic $36 \mathrm{~cm}$ & 1 \\
\hline Suction irrigator & 2 \\
\hline Endoscopic catch bag $15 \mathrm{~mm}$ & 1 \\
\hline Endoscopic stapler $30 \mathrm{~mm}$ & 2 \\
\hline Endoscopic stapler $60 \mathrm{~mm} \times 3.8 \mathrm{~mm}$ & 2 \\
\hline Endoscopic stapler reload $45 \mathrm{~mm} \times 2.5 \mathrm{~mm}$ & 2 \\
\hline Endoscopic stapler reload $60 \mathrm{~mm} \times 3.8 \mathrm{~mm}$ & 1 \\
\hline Endoscopic shear $5 \mathrm{~mm} \times 35 \mathrm{~mm}$ & 1 \\
\hline Grasper $5 \mathrm{~mm} \times 35 \mathrm{~mm}$ & 2 \\
\hline Endoscopic clip applier & 1 \\
\hline Skin stapler $35 \mathrm{~mm}$ wide & 1 \\
\hline Endoscopic trocar blunt $12 \mathrm{~mm} \times 100 \mathrm{~mm}$ & 3 \\
\hline Endoscopic trocar $5 \mathrm{~mm} \times 100 \mathrm{~mm} \mathrm{FIOS}^{\circledR}$ & 2 \\
\hline Endoscopic trocar threaded $15 \mathrm{~mm} \times 100 \mathrm{~mm}$ & 1 \\
\hline Foam pad elbow protector & 1 \\
\hline Feeding tube (available 4-6-8 French) & 2 \\
\hline Surgical drains $15 \mathrm{Fr}$ & 2 \\
\hline Surgical clips large and medium & 2 \\
\hline Ligasure Maryland $37 \mathrm{~cm}$ & 1 \\
\hline Endoscopic clip III 5 mm clip applier & 1 \\
\hline Endostich sofsilk 2-0 48 inches & 8 \\
\hline Prolene $5-0$ blue 36 " C-1 needle & 1 \\
\hline Endoloop coated vicryl 18 inches & 1 \\
\hline Surgipro II 4-0 36" CV-23 needle & 2 \\
\hline Suture PDS 4-0 RB-1 & 8 \\
\hline Suture V LOC 3-0 P-14 18” 180 & 2 \\
\hline
\end{tabular}

TLP, total laparoscopic pancreaticoduodenectomy.

perform a TLP are summarized in Table 1.

\section{Role of team members}

TLP is considered to be among the most complex abdominal surgeries and requires significant amount of technological and human resources both during the operative procedure as well as during the recovery phase.
The procedure requires one surgeon, who will direct the execution of the operation, and two surgical assistants, a first assistant to help with the performance of the various steps of the operation, and a second assistant dedicated to maneuvering the laparoscope. The role of the anesthesiology is of fundamental importance to ensure proper anesthesia, timely monitoring of all physiology parameters, and prompt response in case of unexpected blood loos. The operative team is also composed of one scrub nurse and a circulating nurse. The postoperative role of an expert nutritionist, glucose management nurse, and an endocrinologist expert in diabetes cannot be overemphasized may the needs for insulin therapy arise following pancreatic resection. The nutritionist will guide the patient through the alimentary adjustments needed and will optimized the use of pancreatic enzymes replacement therapy to the need of the specific patients. The glucose management nurse and the endocrinologist will provide the education and the therapeutic expertise necessary to achieve safe satisfactory glycemic control.

\section{Postoperative management}

Once the procedure is completed, the patient is usually extubated in the operating room (OR) and transferred directly to intensive care unit where he or her will remain for less than 24 hrs. During the initial recovery phase the attention is mainly focused on obtaining appropriate fluid resuscitation, pain control and optimal glucose level (i.e., $<180 \mathrm{mg} / \mathrm{dL}$ ). On postoperative day (POD) 1 , the patient is transferred to a regular surgical ward, continuous telemetry monitoring is ensured, and ambulation is strongly encouraged. A post-pancreatectomy diet, consisting of small, frequent, low-fat, high-carbohydrate and -protein meals, can usually be started on POD 4. A detailed summary of the authors' postoperative management approach is provided in Table 2. In the absence of severe complications, the patient can be discharged from the hospital as soon as POD 6.

\section{Tips, tricks, and pitfalls}

- Excellent pre-operative imaging is required as palpating aberrant arterial anatomy is not possible with the laparoscopic approach. Having knowledge of this anatomy will allow success with even aberrant arterial anatomy;

- Complete dissection of the common and hepatic artery 


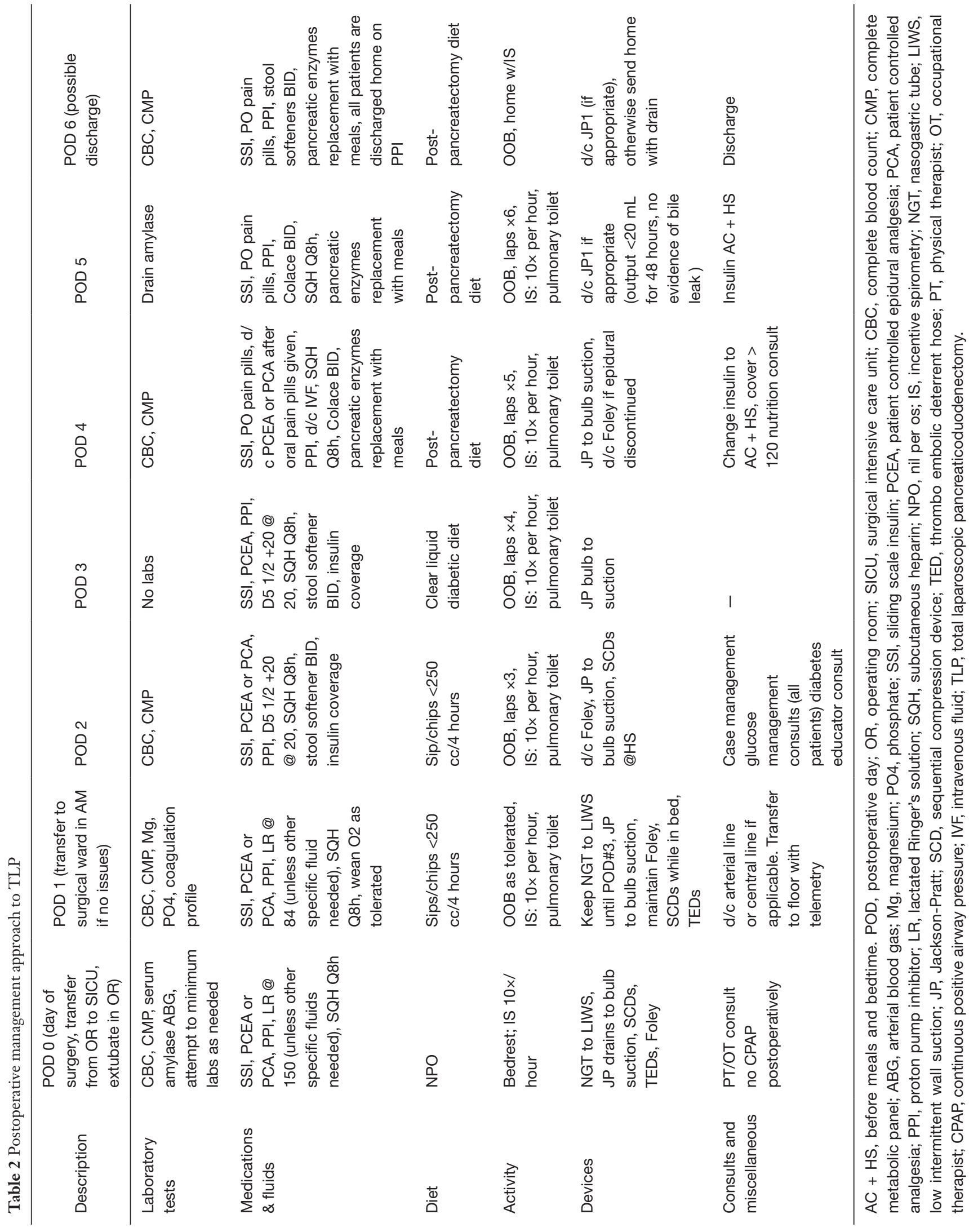


is required prior to transection of the GDA as test occlusion of the GDA and palpation of the proper hepatic is not possible;

- If visualization of the bile duct for the hepaticojejunostomy is difficult, using a looped suture around the base of a mobilized falciform ligament through a poke incision at the base of the xiphoid can lift the liver;

- Bringing the jejunum through the ligament Treitz vs. the meso-colon avoids twisting that can be overlooked laparoscopically and provides a tension free loop for reconstruction;

- Having a laparoscopic bulldog set with various sizes is useful to quickly control bleeding from the portal vein allowing for laparoscopic repair if necessary.

\section{Acknowledgements}

None.

\section{Footnote}

Conflicts of Interest: The authors have no conflicts of interest to declare.

\section{References}

1. Gagner M, Pomp A. Laparoscopic pylorus-preserving pancreatoduodenectomy. Surg Endosc 1994;8:408-10.

2. Paniccia A, Schulick RD, Edil BH. Total Laparoscopic Pancreaticoduodenectomy: A Single-Institutional Experience. Ann Surg Oncol 2015;22:4380-1.

3. Merkow J, Paniccia A, Edil BH. Laparoscopic pancreaticoduodenectomy: a descriptive and comparative review. Chin J Cancer Res 2015;27:368-75.

4. Boggi U, Amorese G, Vistoli F, et al. Laparoscopic pancreaticoduodenectomy: a systematic literature review. Surg Endosc 2015;29:9-23.

5. Song KB, Kim SC, Hwang DW, et al. Matched CaseControl Analysis Comparing Laparoscopic and Open Pylorus-preserving Pancreaticoduodenectomy in Patients With Periampullary Tumors. Ann Surg 2015;262:146-55.

6. Kim SC, Song KB, Jung YS, et al. Short-term clinical outcomes for 100 consecutive cases of laparoscopic pylorus-preserving pancreatoduodenectomy: improvement with surgical experience. Surg Endosc 2013;27:95-103.

7. Speicher PJ, Nussbaum DP, White RR, et al. Defining the learning curve for team-based laparoscopic pancreaticoduodenectomy. Ann Surg Oncol 2014;21:4014-9.

8. Asbun HJ, Stauffer JA. Laparoscopic vs open pancreaticoduodenectomy: overall outcomes and severity of complications using the Accordion Severity Grading System. J Am Coll Surg 2012;215:810-9.

9. Mesleh MG, Stauffer JA, Bowers SP, et al. Cost analysis of open and laparoscopic pancreaticoduodenectomy: a single institution comparison. Surg Endosc 2013;27:4518-23.

10. Croome KP, Farnell MB, Que FG, et al. Total laparoscopic pancreaticoduodenectomy for pancreatic ductal adenocarcinoma: oncologic advantages over open approaches? Ann Surg 2014;260:633-8; discussion 638-40.

11. Croome KP, Farnell MB, Que FG, et al.

Pancreaticoduodenectomy with major vascular resection: a comparison of laparoscopic versus open approaches. J Gastrointest Surg 2015;19:189-94; discussion 194.

12. Drymousis P, Raptis DA, Spalding D, et al. Laparoscopic versus open pancreas resection for pancreatic neuroendocrine tumours: a systematic review and metaanalysis. HPB (Oxford) 2014;16:397-406.

13. Kooby DA, Hawkins WG, Schmidt CM, et al. A multicenter analysis of distal pancreatectomy for adenocarcinoma: is laparoscopic resection appropriate? J Am Coll Surg 2010;210:779-85, 786-7.

14. Mabrut JY, Fernandez-Cruz L, Azagra JS, et al. Laparoscopic pancreatic resection: results of a multicenter European study of 127 patients. Surgery 2005;137:597-605.

15. Nakamura M, Wakabayashi G, Miyasaka Y, et al. Multicenter comparative study of laparoscopic and open distal pancreatectomy using propensity score-matching. J Hepatobiliary Pancreat Sci 2015;22:731-6.

16. Langan RC, Graham JA, Chin AB, et al. Laparoscopicassisted versus open pancreaticoduodenectomy: early favorable physical quality-of-life measures. Surgery 2014;156:379-84.

17. Adam MA, Choudhury K, Dinan MA, et al. Minimally Invasive Versus Open Pancreaticoduodenectomy for Cancer: Practice Patterns and Short-term Outcomes Among 7061 Patients. Ann Surg 2015;262:372-7.

18. Sharpe SM, Talamonti MS, Wang CE, et al. Early National Experience with Laparoscopic Pancreaticoduodenectomy for Ductal Adenocarcinoma: A Comparison of Laparoscopic Pancreaticoduodenectomy and Open Pancreaticoduodenectomy from the National Cancer Data Base. J Am Coll Surg 2015;221:175-84. 
19. Kendrick ML, Sclabas GM. Major venous resection during total laparoscopic pancreaticoduodenectomy. HPB (Oxford) 2011;13:454-8.

20. Paniccia A, Chapman B, Schulick RD, et al. The

doi: 10.21037/jovs.2016.07.13

Cite this article as: Paniccia A, Chapman B, Edil BH, Schulick

RD. Total laparoscopic pancreaticoduodenectomy. J Vis Surg 2016;2:130. video illustrates the key steps performed during a total laparoscopic pancreaticoduodenectomy. Asvide 2016;3:295. Available online: http://www.asvide.com/ articles/1057 